\title{
Evidence for metastable photo-induced superconductivity in $\mathrm{K}_{3} \mathrm{C}_{60}$
}

\author{
M. Budden' ${ }^{1 凶}$, T. Gebert', M. Buzzi ${ }^{1}$ ', G. Jotzu $\oplus^{1}$, E. Wang', T. Matsuyama', G. Meier ${ }^{1}$, Y. Laplace',

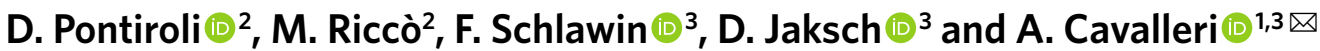

Excitation of high- $T_{c}$ cuprates and certain organic superconductors with intense far-infrared optical pulses has been shown to create non-equilibrium states with optical properties that are consistent with transient high-temperature superconductivity. These non-equilibrium phases have been generated using femtosecond drives, and have been observed to disappear immediately after excitation, which is evidence of states that lack intrinsic rigidity. Here we make use of a new optical device to drive metallic $\mathrm{K}_{3} \mathrm{C}_{60}$ with mid-infrared pulses of tunable duration, ranging between one picosecond and one nanosecond. The same superconducting-like optical properties observed over short time windows for femtosecond excitation are shown here to become metastable under sustained optical driving, with lifetimes in excess of ten nanoseconds. Direct electrical probing, which becomes possible at these timescales, yields a vanishingly small resistance with the same relaxation time as that estimated by terahertz conductivity. We provide a theoretical description of the dynamics after excitation, and justify the observed slow relaxation by considering randomization of the order-parameter phase as the rate-limiting process that determines the decay of the light-induced superconductor.

$\mathrm{N}$ on-equilibrium orders in complex materials include photo-induced ferroelectricity ${ }^{1,2}$, magnetic polarization in antiferromagnets ${ }^{3}$ and transient superconductivity in the normal state of cuprates and organic conductors ${ }^{4-9}$. Among these, much work has been dedicated to alkali-doped fullerides of the $\mathrm{A}_{3} \mathrm{C}_{60}$ family (Fig. 1a), which exhibit tunable high-temperature superconductivity at equilibrium ${ }^{10-15}$ (Fig. 1b). The dynamical manipulation of superconductivity in these materials ${ }^{6,7}$ has been demonstrated by using optical pulses at mid-infrared frequencies that are tuned in the vicinity of local vibrational resonances of the $\mathrm{C}_{60}$ molecules.

The evidence reported so far is summarized in Fig. $1 c, d . \mathrm{K}_{3} \mathrm{C}_{60}$ powders were held at a base temperature of $T=100 \mathrm{~K} \gg T_{\mathrm{c}}=20 \mathrm{~K}$ and irradiated with 100-fs-long, 7.3- $\mu \mathrm{m}$-wavelength $(\hbar \omega \approx 170 \mathrm{meV}$, where $\hbar$ is Planck's constant and $\omega$ is angular frequency) pulses at a fluence of $3 \mathrm{~mJ} \mathrm{~cm} \mathrm{~cm}^{-2}$, which yielded a short-lived transient state with large changes in the terahertz optical properties. The transient optical response was probed with phase-sensitive, time-domain terahertz spectroscopy, which directly yields the real and imaginary part of the reflectance and hence can be used to retrieve the complex optical conductivity without the need of Kramers-Kronig transformations (Supplementary Section 4 and refs. ${ }^{6,7}$ ). The transient low-frequency optical conductivity induced by femtosecond excitation was almost indistinguishable from that of the equilibrium superconducting state measured in the same material at $T \ll T_{\mathrm{c}}=20 \mathrm{~K}$ (compare to Fig. 1c). Such 'superconducting-like' optical properties consist of a perfect low-energy reflectance $(R=1)$, a vanishingly small real part of the optical conductivity $\sigma_{1}(\omega)$ for all photon energies lower than the energy gap $2 \Delta$ and an imaginary conductivity $\sigma_{2}(\omega)$ that diverges toward low frequencies as $1 / \omega$, which itself is indicative of a large zero-frequency conductivity.

These observations have generated interest, as they may make it possible to achieve photo-induced superconducting states ${ }^{4-7,9,16,17}$ at or in the vicinity of room temperature. However, all of the experiments reported have indicated the presence of states that disappear immediately after optical excitation (see optical properties measured at 5 ps time delay in Fig. 1d). These short lifetimes would prevent most applications and have even raised controversy over the data interpretation itself ${ }^{18,19}$.

In this Article, we explore the possibility of longer-lived superconductivity under a sustained optical drive. First, we modified the experimental set-up used for the experiments shown in Fig. 1 and lengthened the $7.3 \mu \mathrm{m}$ wavelength pump pulses by making them propagate in a dispersive $\mathrm{CaF}_{2}$ rod (Fig. 2a). These chirped pump pulses had a duration $\tau_{\mathrm{p}} \approx 1 \mathrm{ps}$, which enabled a sixfold increase in the pulse energy density (up to $18 \mathrm{~mJ} \mathrm{~cm}^{-2}$ ) without a corresponding increase in the peak electric field. This longer pulse duration allowed us to conduct these experiments at pulse energy densities that would damage the sample at femtosecond pulse durations, and hence it enabled the exploration of a new regime of excitation.

The pump-induced changes in the low-frequency reflectivity and complex optical conductivity (Fig. 2b) were measured on the same sample and at the same temperature $T=100 \mathrm{~K}$ as for the experiments of Fig. 1. Representative reflectivity spectra $R(\omega)$ and complex optical conductivity, $\sigma_{1}(\omega)+i \sigma_{2}(\omega)$, measured for a 1 ps pump-pulse duration and $18 \mathrm{~mJ} \mathrm{~cm}^{-2}$ fluence are reported for time delays of -5 ps, 10 ps, 300 ps and 12 ns. These plots indicate a similar response to that shown in Fig. 1d (perfect reflectivity $(R=1)$, gapped $\sigma_{1}(\omega)$ and a divergent $\left.\sigma_{2}(\omega)\right)$, although with a far longer relaxation time of at least 300 ps.

A more comprehensive exploration of sustained optical driving, beyond the limited pulse-width tunability of the set-up shown in Fig. 2, is shown in Figs. 3, 4 and 5. These measurements made use of a newly developed optical device, based on a $\mathrm{CO}_{2}$-gas laser that was optically synchronized to a femtosecond $\mathrm{Ti}: \mathrm{Al}_{2} \mathrm{O}_{3}$ laser, and that delivered $10.6 \mu \mathrm{m}$ wavelength pulses with durations that could be tuned between 5 ps and 1.3 ns (Fig. 3a).

${ }^{1}$ Max Planck Institute for the Structure and Dynamics of Matter, Hamburg, Germany. ${ }^{2}$ Dipartimento di Scienze Matematiche, Fisiche e Informatiche, Università degli Studi di Parma, Parma, Italy. ${ }^{3}$ Department of Physics, Clarendon Laboratory, University of Oxford, Oxford, UK. 凶e-mail: matthias.budden@mpsd.mpg.de; andrea.cavalleri@mpsd.mpg.de 
a

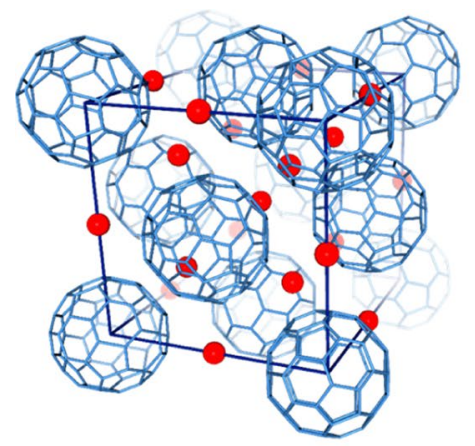

b

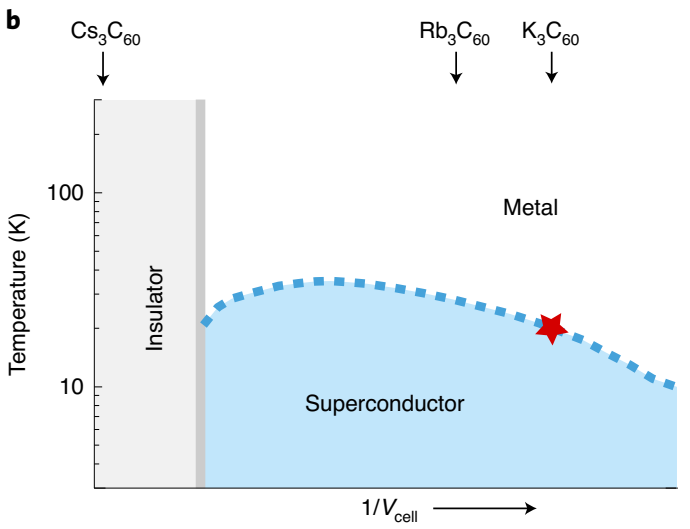

C Equilibrium

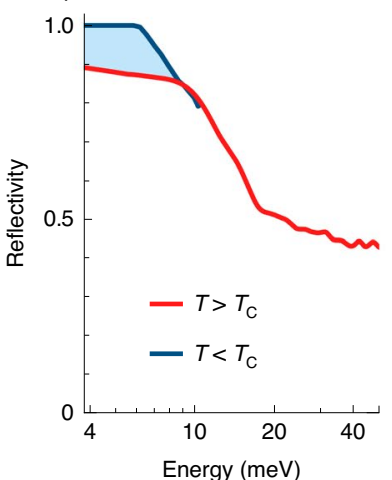

d

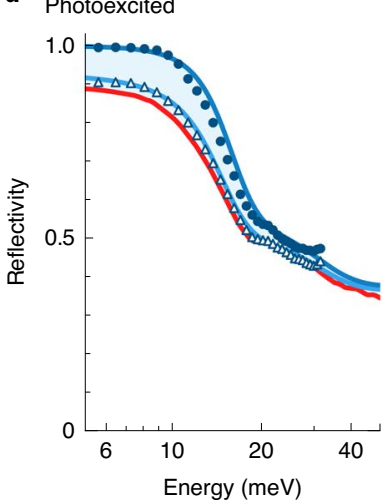

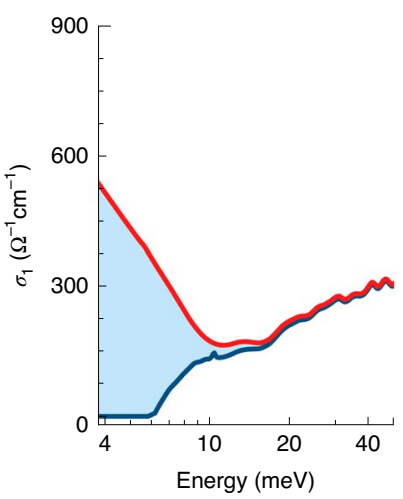
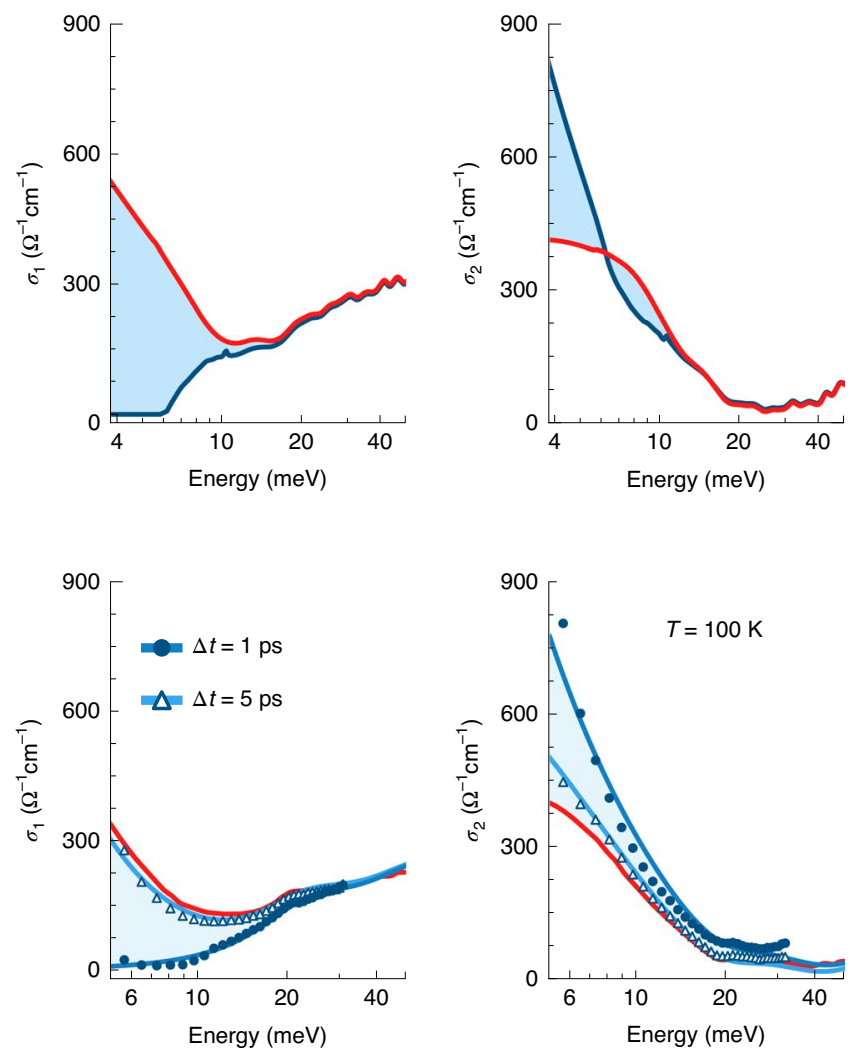

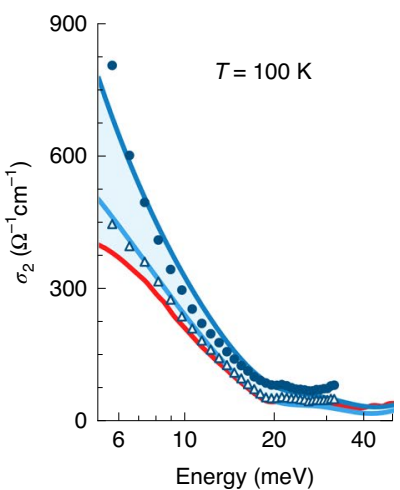

Fig. 1 | Crystal structure, phase diagram, equilibrium phase transition and short-lived, light-induced phase in $\mathrm{K}_{3} \mathrm{C}_{60}$. $\mathbf{a}_{1}$ Crystal structure of the molecular superconductor $\mathrm{K}_{3} \mathrm{C}_{60}$. $\mathrm{C}_{60}$ molecules are arranged in a face-centred cubic lattice. Potassium atoms (red) occupy the interstitial voids. $\mathbf{b}$, Phase diagram of the $\mathrm{A}_{3} \mathrm{C}_{60}$ alkali-doped fullerene family as a function of the inverse unit cell volume $1 / V_{\text {cell }}$. The ground state of the material can be tuned by chemical or physical pressure. The solid grey line indicates the boundary between the insulating and metallic/superconducting phases. The blue dashed line highlights the dependence of the superconducting transition temperature $T_{c}$ as a function of $1 / V_{\text {cell }}$. The star indicates the $\mathrm{K}_{3} \mathrm{C}_{60}$ compound investigated in the present work, which becomes superconducting for $T<T_{c}=20 \mathrm{~K}$. c. Equilibrium reflectivity (sample-diamond interface), real and imaginary part of the optical conductivity of $\mathrm{K}_{3} \mathrm{C}_{60}$ measured upon cooling across the equilibrium superconducting transition. The blue shading indicates the change of spectral weight in these quantities across the thermally driven superconducting transition. d, Same quantities measured at equilibrium (red lines), 1 ps (blue filled dots) and $5 \mathrm{ps}$ (light blue triangles) after photoexcitation. The blue shading indicates the change of spectral weight in these quantities induced by photoexcitation. The light and dark blue lines are Drude-Lorentz fits to the transient optical data (Supplementary Section 6). These data were acquired at a base temperature $T=100 \mathrm{~K}$ with a fluence of $3 \mathrm{~mJ} \mathrm{~cm}^{-2}$ and a pulse duration of $100 \mathrm{fs}$.

Near-infrared femtosecond pulses from a $\mathrm{Ti}: \mathrm{Al}_{2} \mathrm{O}_{3}$ laser were converted to a wavelength of $10.6 \mu \mathrm{m}$ with an optical parametric amplifier and were used to seed a $\mathrm{CO}_{2}$ laser oscillator ${ }^{20}$. The oscillator emitted trains of nanosecond-long pulses, out of which the most intense was selected by a Pockels cell and amplified to an energy of $10 \mathrm{~mJ}$ in a second, multi-pass $\mathrm{CO}_{2}$ laser amplifier. The duration of these amplified pulses was then tuned as shown in Fig. $3 \mathrm{a}$.
The 'front' and the 'back' of the 1-ns-long $\mathrm{CO}_{2}$ pulses were 'sliced' using a pair of photoexcited semiconductor wafers as plasma mirrors $^{21-23}$. As the wafers were set at Brewster's angle and their bandgap was much larger than the $117 \mathrm{meV}$ photon energy of the $\mathrm{CO}_{2}$ laser, these were almost perfectly transparent when unexcited. Pairs of femtosecond optical pulses struck each wafer at adjustable time delays, making these reflective owing to the injection of dense 
a

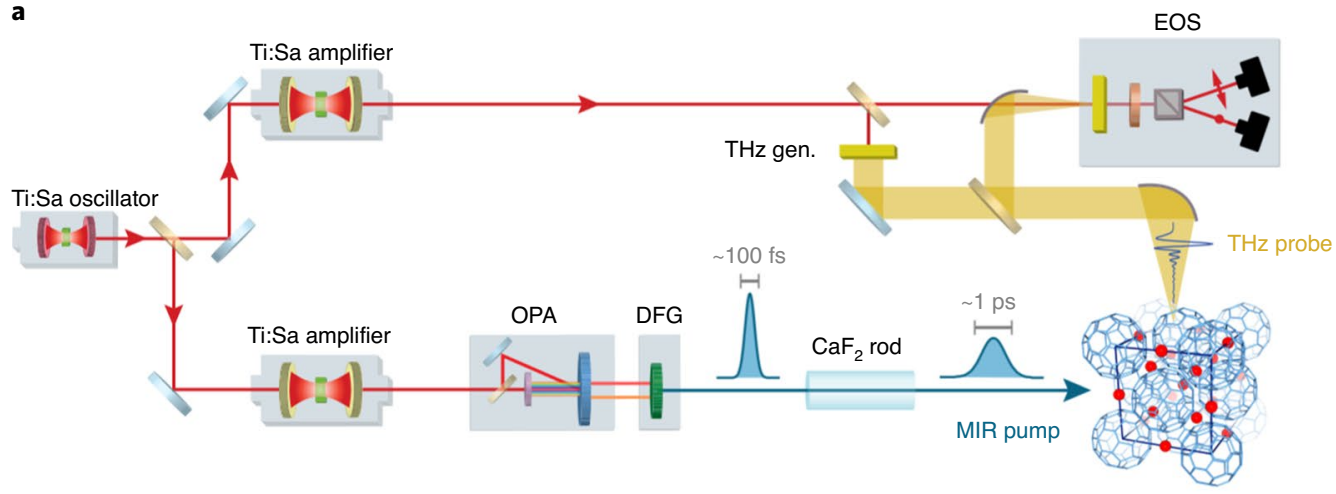

b High-fluence photoexcitation
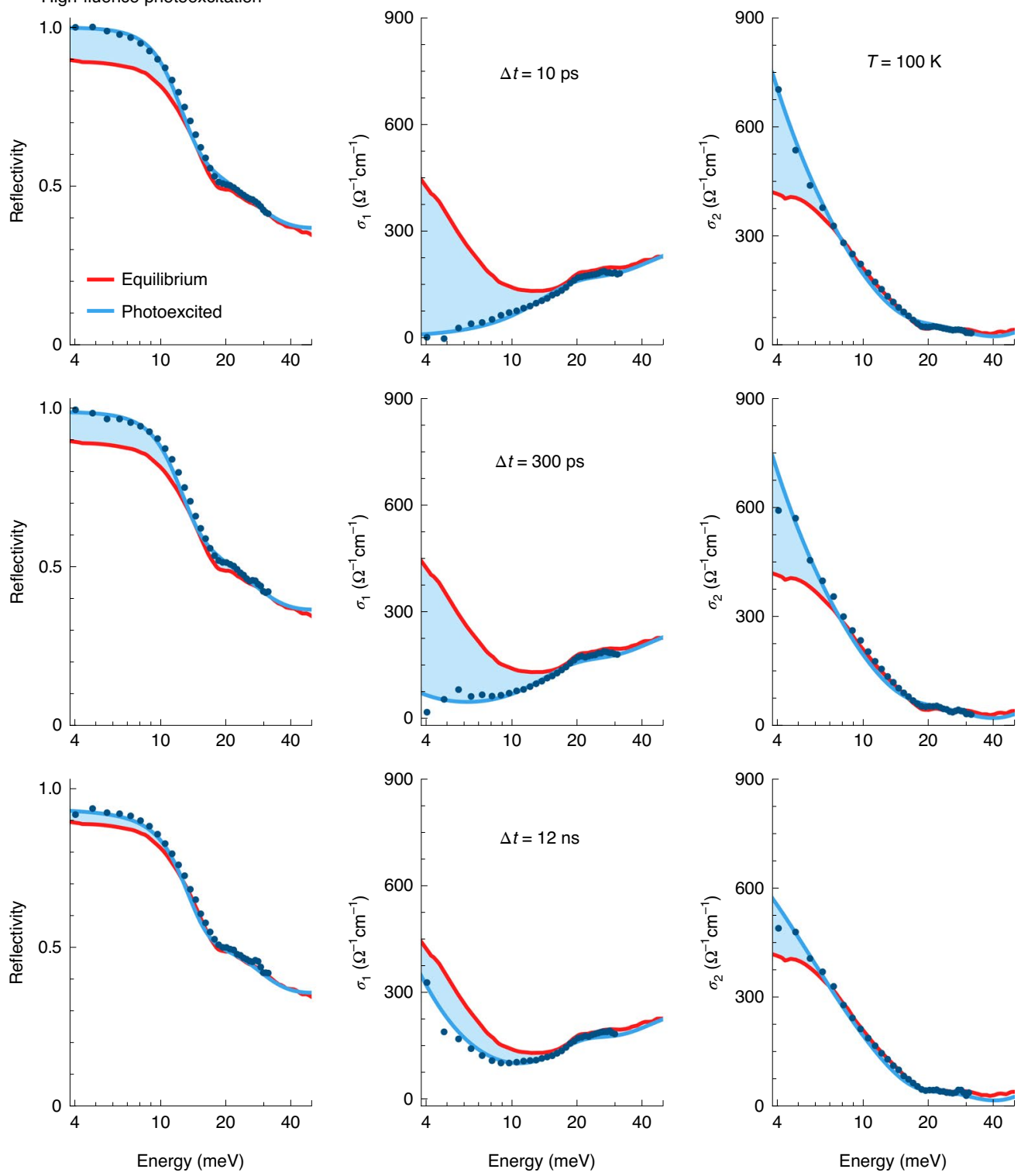

Fig. 2 | Long-lived, light-induced phase in $\mathrm{K}_{3} \mathrm{C}_{60}$ generated with intense, 1-ps-long excitation pulses. a, Schematic of the experimental set-up for pump-probe experiments producing 1-ps-long pump pulses centred at a mid-infrared (MIR) wavelength of $7.3 \mu \mathrm{m}$. These pulses have been generated in an optical parametric amplifier (OPA) and subsequent difference frequency generation (DFG) of the signal and idler beams. The 7.3- $\mu$ m-wavelength, 100-fs-long pulses are stretched by linear propagation in a highly dispersive $\mathrm{CaF}_{2}$ rod. The photo-induced changes in the optical response of $\mathrm{K}_{3} \mathrm{C}_{60}$ upon irradiation are detected with transient terahertz time-domain spectroscopy using electro-optic sampling (EOS) for detection of the reflected terahertz field. $\mathbf{b}$, Equilibrium reflectivity (samplediamond interface), real and imaginary part of the optical conductivity of $\mathrm{K}_{3} \mathrm{C}_{60}$ measured at equilibrium (red lines), $10 \mathrm{ps}, 300 \mathrm{ps}$ and $12 \mathrm{~ns}$ (blue filled symbols) after photoexcitation. The blue shading indicates the change of spectral weight in these quantities induced by photoexcitation. The dark blue lines are DrudeLorentz fits to the transient optical data (Supplementary Section 6). These data were acquired at a base temperature $T=100 \mathrm{~K}$ with a fluence of $18 \mathrm{~mJ} \mathrm{~cm}^{-2}$ and a pump-pulse duration of $1 \mathrm{ps}$. 
a

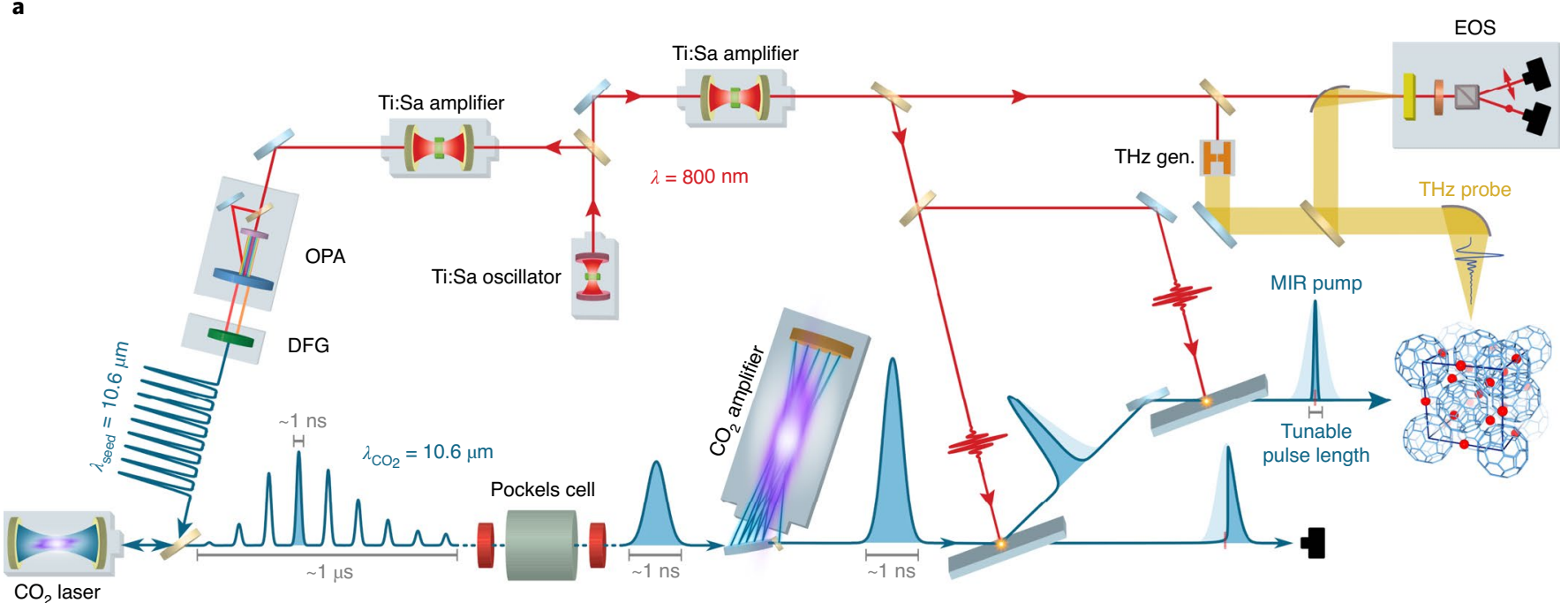

b Long pulses
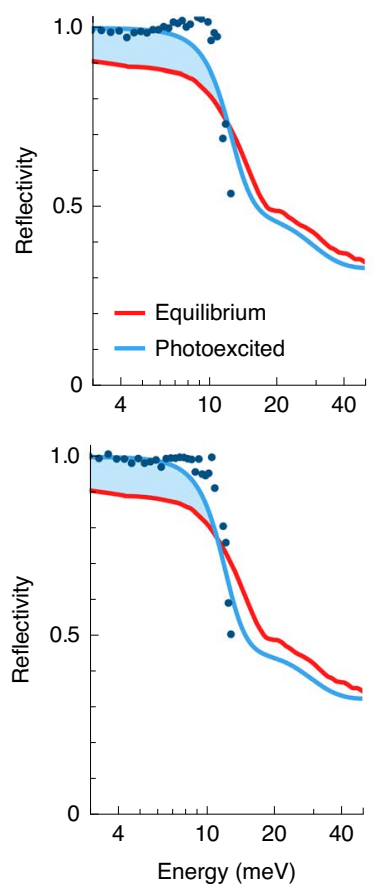
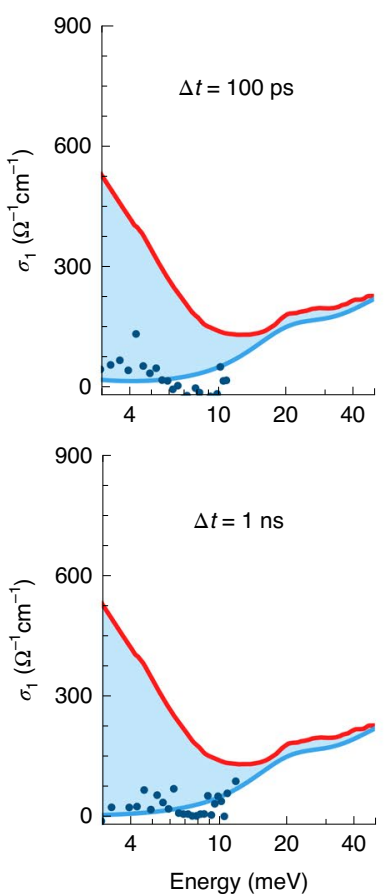

c

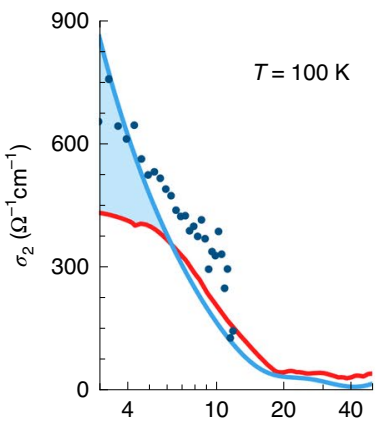

-
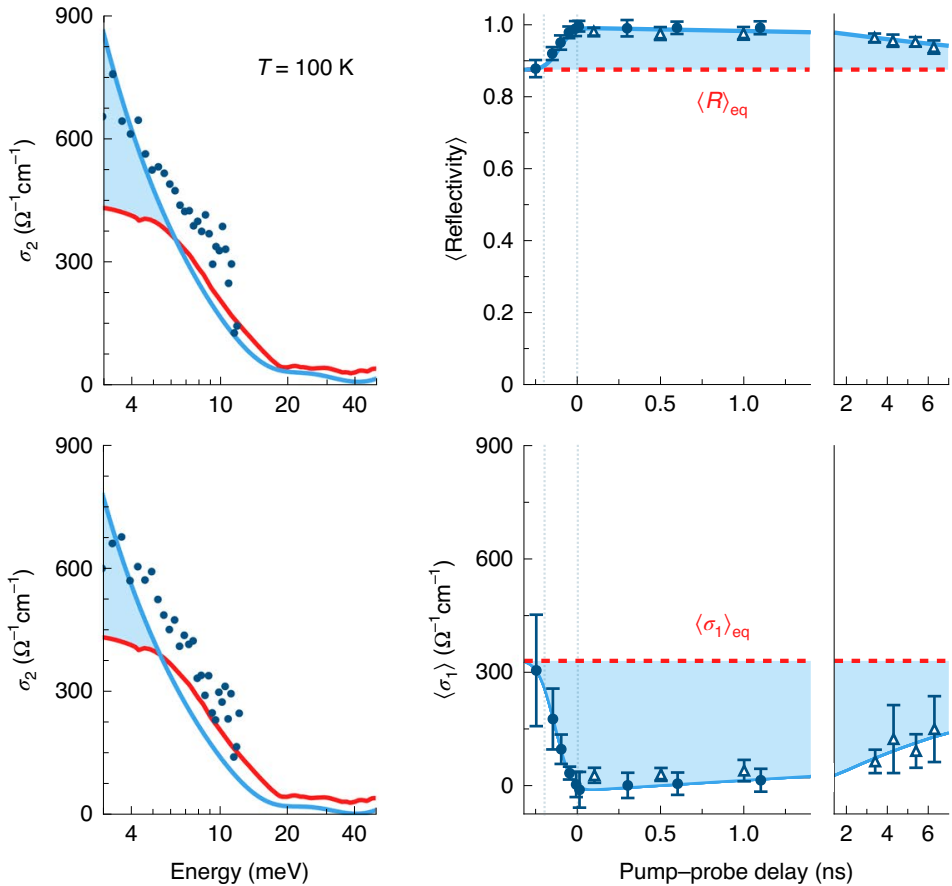

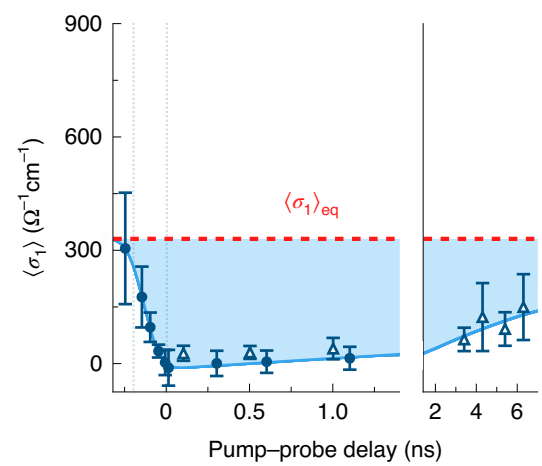

Fig. 3 | Long-lived, light-induced phase in $\mathrm{K}_{3} \mathrm{C}_{60}$ generated with intense, 300-ps-long excitation pulses. a, Schematic of the experimental set-up for pumpprobe experiments that produced picosecond pulses of duration variable between $5 \mathrm{ps}$ and $1.3 \mathrm{~ns}$, centred at $10.6 \mu \mathrm{m}$ wavelength and with a pulse energy of up to $10 \mathrm{~mJ}$. The pulses are generated by seeding a $\mathrm{CO}_{2}$-gas laser with a femtosecond source. After amplification in a second $\mathrm{CO}_{2}$-gas laser, the pulse duration is tuned by semiconductor slicing. The photo-induced changes in the optical response of $\mathrm{K}_{3} \mathrm{C}_{60}$ upon irradiation are detected with transient terahertz time-domain spectroscopy. b, Equilibrium reflectivity (sample-diamond interface), real and imaginary part of the optical conductivity of $\mathrm{K}_{3} \mathrm{C}_{60}$ measured at equilibrium (red lines), $100 \mathrm{ps}$ and $1 \mathrm{~ns}$ (blue filled symbols) after photoexcitation. The dark blue lines are Drude-Lorentz fits to the transient optical data (Supplementary Section 6). These data were acquired at a base temperature $T=100 \mathrm{~K}$ with a fluence of $53 \mathrm{~mJ} \mathrm{~cm} \mathrm{~cm}^{-2}$ and a pump-pulse duration of $300 \mathrm{ps}$. The blue shading indicates the change of spectral weight in these quantities induced by photoexcitation. $\mathbf{c}$, Time dependence of the average reflectivity and real part of the optical conductivity $\sigma_{1}(\omega)$ in the region of the photo-induced gap (2-10 meV). These data were acquired at a base temperature $T=100 \mathrm{~K}$ with a fluence of $25 \mathrm{~mJ} \mathrm{~cm}^{-2}$ and a pump-pulse duration of $200 \mathrm{ps}$. Triangles and circles denote measurements on two different samples. The top panel shows the measured time profile of the excitation pulse. Error bars represent the standard deviation of the respective quantity over the averaged range. The red dashed lines represent the equilibrium average value of $\sigma_{1}(\omega)$ at equilibrium. The blue shading indicates the change of $\left\langle\sigma_{1}(\omega)\right\rangle$ from the equilibrium value after photoexcitation.

electron hole plasmas. Pump pulses with a duration tunable from 5 ps to $1.3 \mathrm{~ns}$ were generated in this way and used to pump the $\mathrm{K}_{3} \mathrm{C}_{60}$ sample (Supplementary Section 3), which was probed with the same time-domain terahertz reflectivity probe used for the experiments of Figs. 1 and 2.
Figure $3 \mathrm{~b}$ displays snapshots of the transient optical properties $\left(R(\omega), \sigma_{1}(\omega), \sigma_{2}(\omega)\right)$ measured for $\mathrm{K}_{3} \mathrm{C}_{60}$ at $T=100 \mathrm{~K}$ before photoexcitation and 100 ps and $1 \mathrm{~ns}$ after photoexcitation with a 300-ps-long pulse centred at a wavelength of $10.6 \mu \mathrm{m}$. As these measurements were obtained for a repetition rate of $18 \mathrm{~Hz}$, the signal-to-noise ratio 

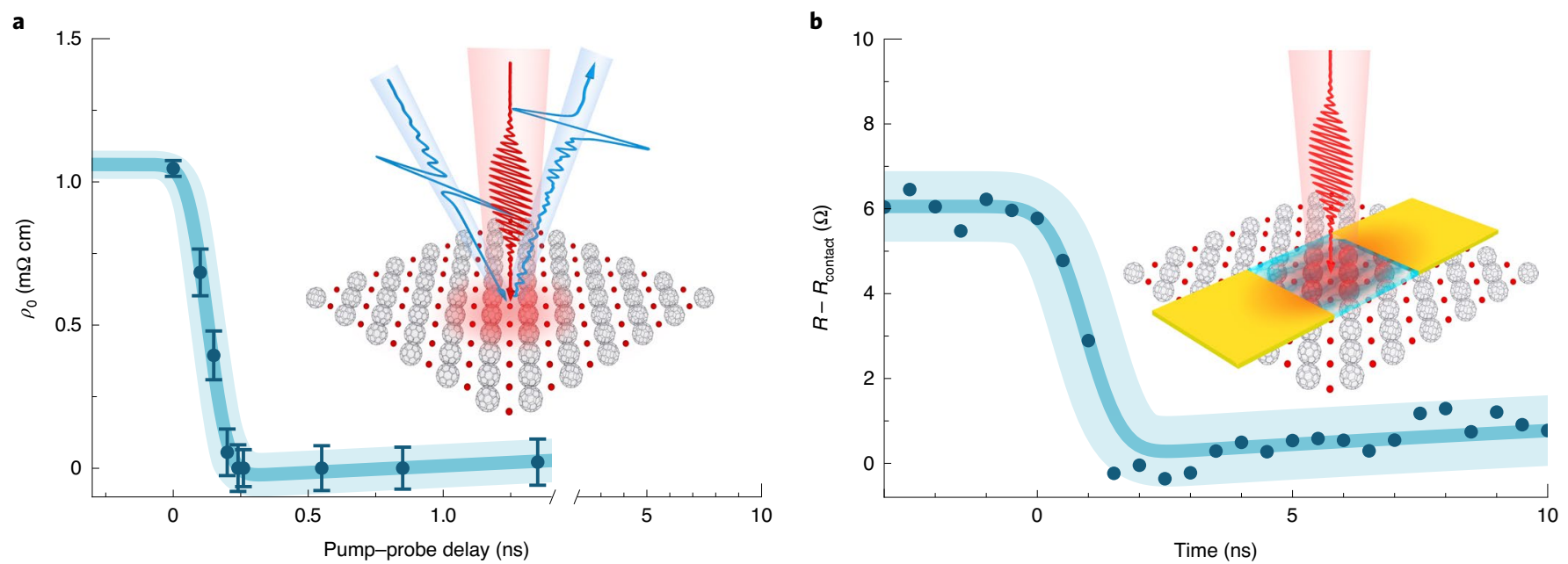

Fig. 4 | Transient resistivity measurements of the long-lived, light-induced phase in $\mathrm{K}_{3} \mathbf{C}_{60}$. $\mathbf{a}$, Time evolution of the transient resistivity $\rho_{0}$ obtained from an extrapolation to zero frequency of a Drude-Lorentz fit to the transient optical conductivities (Supplementary Section 6). Error bars represent the $\rho_{0}$ confidence interval obtained from the fit. The inset shows a schematic depiction of the experimental arrangement indicating pump and probe beam and their electric fields in red and blue, respectively. $\mathbf{b}$, Resistance of a laser-irradiated $\mathrm{K}_{3} \mathrm{C}_{60}$ pellet that was embedded in a microstrip transmission line as a function of time after photoexcitation. The resistance value is obtained from a transient two-point transport measurement in which contributions from contact resistances are calibrated from a static four-point measurement. The shaded area indicates an estimate of the systematic error that is introduced by this calibration (see the main text and Supplementary Sections 7-9). The inset shows a schematic of the electrical transport experiment with gold electrodes on $\mathrm{K}_{3} \mathrm{C}_{60}$ in yellow and the region of current flow in teal. The pump beam and its electric field are displayed in red. These data were acquired at a base temperature $T=100 \mathrm{~K}$ with a fluence of $\sim 25 \mathrm{~mJ} \mathrm{~cm} \mathrm{~cm}^{-2}$. The pump-pulse durations were $200 \mathrm{ps}$ and $75 \mathrm{ps}$ for the optical and transport measurements, respectively.

is reduced compared with that of the cases shown in Figs. 1 and 2, which were measured at $500 \mathrm{~Hz}$.

The transient optical spectra measured in these conditions showed the same superconducting-like features as reported in Figs. 1d and $2 \mathrm{~b}$ for all pump-pulse durations up to $1 \mathrm{~ns}$ after excitation. Note that the $10.6 \mu \mathrm{m}$ wavelength radiated by the $\mathrm{CO}_{2}$ laser is different from the $7.3 \mu \mathrm{m}$ wavelength used in the experiments of Figs. 1 and 2. However, excitation with femtosecond optical pulses at this wavelength had previously been shown to induce the same transient optical signatures generated with $7.3 \mu \mathrm{m}$ wavelength excitation, although with a lower efficiency ${ }^{6}$.

The time evolution of the terahertz optical properties is shown in Fig. 3c. The top panel shows the average reflectivity $R(\omega)$ in the region where $\sigma_{1}(\omega)$ exhibited a gap $(2-10 \mathrm{meV})$. The lower panel shows the average value of the corresponding real part of the optical conductivity $\sigma_{1}(\omega)$, which reached zero after optical excitation, reflecting full gapping. Both quantities remained unchanged after excitation for all time delays measured up to $1 \mathrm{~ns}$. Extended measurements indicate a lifetime of the light-induced superconducting state of $\tau_{\mathrm{d}} \approx 10 \mathrm{~ns}$ (Supplementary Section 11 ).

From the optical spectra, we also extracted an estimate of the 'zero-frequency resistivity' $\rho_{0}=1 / \lim _{\omega \rightarrow 0} \sigma_{1}(\omega)$, which is based on a Drude-Lorentz fit to the transient optical properties (Supplementary Section 6). This fitting procedure yielded a vanishingly small $\rho_{0}(t)$ for all time delays after excitation (Fig. 4a).

Estimates of $\rho_{0}$ from optical measurements were complemented with direct electrical measurements. The $\mathrm{K}_{3} \mathrm{C}_{60}$ pellets were incorporated into lithographically patterned microstrip transmission lines. Their resistance was tracked at different times after excitation by transmitting a $1 \mathrm{~ns}$ voltage probe pulse that yielded time-resolved two-terminal resistance measurements (Supplementary Sections 7 and 8). The contributions due to contact resistance were normalized by performing equilibrium four-terminal measurements that were subtracted from the time-resolved resistance measurements (Supplementary Section 8).
Figure $4 \mathrm{~b}$ shows the time evolution of the two-terminal resistance of a $\mathrm{K}_{3} \mathrm{C}_{60}$ pellet measured at $T=100 \mathrm{~K}$ upon photoexcitation in similar conditions to those of the optical experiments reported in Fig. 3. As seen in the resistivity extrapolated from the optical measurements (compare to Fig. 4a), upon excitation the resistance drops to a value that is compatible with zero and recovers on the same timescale of tens of nanoseconds that was extracted from the fitted results of Fig. 4a (Supplementary Section 11).

The electrical probe experiments were repeated by varying the excitation pulse duration and fluence. Figure 5 a shows exemplary pump-pulse duration dependencies of the measured sample pho-

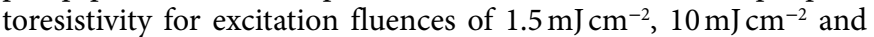
$25 \mathrm{~mJ} \mathrm{~cm}^{-2}$. The photoresistivity was mostly independent of the pump-pulse duration and depended only on the total energy of the excitation pulse. This is also underscored by the data shown in Fig. 5b, which illustrates the dependence of the sample resistance on the excitation fluence at a constant pulse duration. A long-lived state featuring zero resistance was observed for all excitation fluences in excess of $20 \mathrm{~mJ} \mathrm{~cm}^{-2}$.

These data provide evidence for a metastable state of $\mathrm{K}_{3} \mathrm{C}_{60}$ with a very large positive photoconductivity. The observation of such a large and positive photoconductivity would be highly unconventional for a metal, which generally exhibits photoconductivities of less than $1 \%$ (refs. ${ }^{24-26}$ ) and which are often negative ${ }^{27}$, especially when excitation is performed in the mid-infrared and far away from inter-band transitions. Rather, the combined observation of a vanishingly small resistance with the transient terahertz optical spectra shown in Fig. 3 substantiates the assignment of a metastable superconducting state.

Note that the photo-induced high-temperature state survives far longer than the drive pulse, and hence exhibits intrinsic rigidity at timescales when the coherence is no longer supplied by the external drive. In search for a mechanism behind this long lifetime, we applied a phenomenological time-dependent Ginzburg-Landau model to capture the dynamics of the superconducting order parameter after laser excitation. In this model, we did not address the microscopic 
a

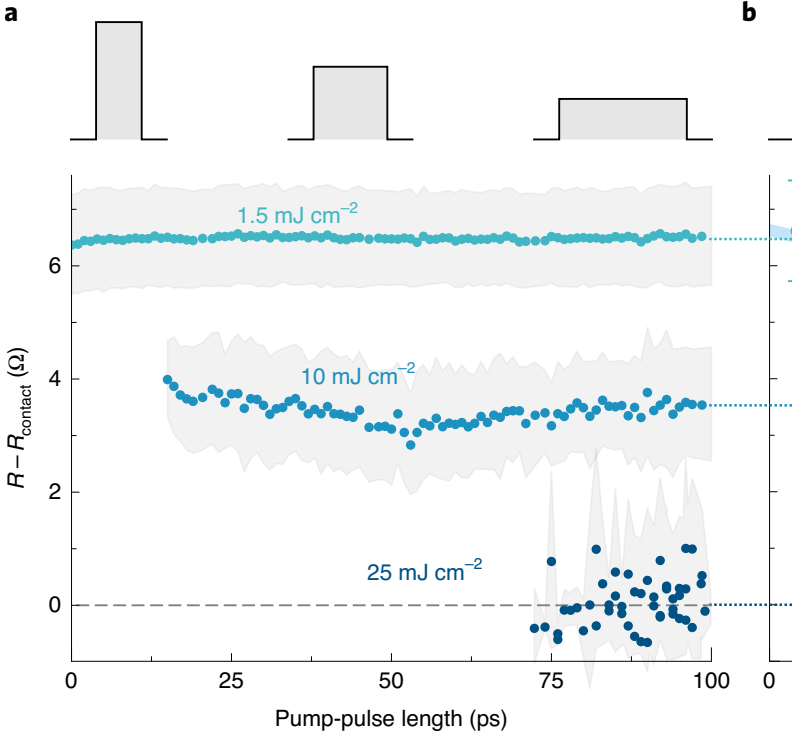

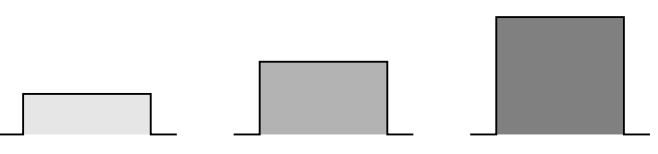

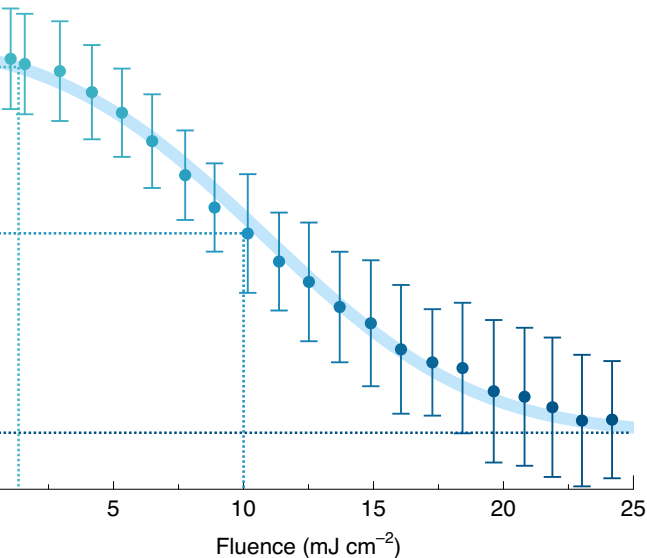

Fig. 5 | Pump-pulse duration and fluence dependence of the long-lived, light-induced phase in $\mathbf{K}_{3} \mathbf{C}_{60}$. $\mathbf{a}$, Pulse-length dependence of the resistance of a laser-irradiated $\mathrm{K}_{3} \mathrm{C}_{60}$ pellet measured at three different representative fluences of $1.5 \mathrm{~mJ} \mathrm{~cm}^{-2}, 10 \mathrm{~mJ} \mathrm{~cm}^{-2}$ and $25 \mathrm{~mJ} \mathrm{~cm}^{-2}$. For the higher fluence values of $10 \mathrm{~mJ} \mathrm{~cm}^{-2}$ and $25 \mathrm{~mJ} \mathrm{~cm}^{-2}$, the minimum achievable pulse length is higher owing to limitations of our experimental set-up. Resistance values were measured right after the end of the pump pulse. The shading represents the standard deviation calculated on average from a set of 300,70 and 10 measurements per point, respectively. The sample resistance was obtained as in Fig. 4 by calibrating the contributions of contact resistance in the transient two-terminal measurement from a static four-point measurement (Supplementary Section 8 ). The dashed grey line highlights $R-R_{\text {contact }}=0$. b, Corresponding fluence dependence of the calibrated sample resistance measured at a constant pump-pulse duration of 95 ps, with dotted lines identifying the different fluence values. The solid blue curve is a guide to the eye. The error bars display the standard deviation of the mean. The top panels schematically show how the shape of the excitation pulse evolves as function of their duration (a) and fluence (b). The shading represents the excitation fluence with darker shades of grey indicating a larger fluence. These data were measured at a base temperature $T=100 \mathrm{~K}$.

mechanism for the formation of superconducting order, but posited its emergence under optical excitation for a base temperature far in excess of the equilibrium transition temperature $T_{c}$. As argued in ref. ${ }^{28}$, the normal state of unconventional superconductors that are susceptible to being ordered with light may be that of a phase-incoherent bosonic fluid, in which superconducting fluctuations are present already. Hence, we consider a hypothetical situation in which phase synchronization is established by a light field, and we study how one such order is lost after the driving pulse has been turned off.

In our time-dependent Ginzburg-Landau simulations, a local superconducting order parameter $\psi_{m}(t)=\left|\psi_{m}\right| e^{i \varphi_{m}}$ was assigned on each lattice site. The average of these wave functions through the whole lattice $\left\langle\psi_{m}\right\rangle$ described the macroscopic properties of the system (Supplementary Section 13). The relaxation of a superconducting state $\left(\left|\left\langle\psi_{m}\right\rangle\right|>0\right)$ to the equilibrium metallic one $\left(\left|\left\langle\psi_{m}\right\rangle\right|=0\right)$ can happen either by a fast decrease of the amplitudes of the local order parameter $\left|\psi_{m}\right|$ (that is, by annihilation of Cooper pairs) or by randomization of the order-parameter phase $\varphi_{m}$. By construction, the latter dominates in a phase-incoherent superconductor, in which phase fluctuations are considerably larger than the amplitude ones. In this case, the local free energy surface shows a minimum, even above $T_{c}$, at a finite local order-parameter amplitude $\left|\psi_{m}\right|$ and suppresses amplitude fluctuations (Fig. 6a). We found that, whereas the relaxation to the non-superconducting ground state occurs by thermally driven diffusion of the local phases, the synchronized state can survive considerably longer than that observed in many cases that consider amplitude relaxation only ${ }^{29,30}$.

Figure $6 \mathrm{~b}$ displays the time dependence of the integrated spectral weight loss over the optical gap $\Delta \sigma_{1}=\int\left[\sigma_{1}^{\text {equil }}(\omega)-\sigma_{1}^{\text {trans }}(\omega)\right] \mathrm{d} \omega$, a quantity that is proportional to the superfluid density in a superconductor. Here, $\sigma_{1}^{\text {trans }}(\omega)$ and $\sigma_{1}^{\text {equil }}(\omega)$ are the real parts of the optical conductivities measured in the photo-induced and equilibrium state, respectively. This quantity can be compared to the normalized amplitude of the averaged order parameter $\left|\psi_{m}\right|$ that is extracted from the time-dependent Ginzburg-Landau simulations (Supplementary Section 13), yielding a relaxation time of $\sim 12 \mathrm{~ns}$. Figure $6 \mathrm{c}-\mathrm{e}$ complements this observation and shows the time evolution of the local complex order parameters $\psi_{m}$ that were extracted from the same simulations $0 \mathrm{~ns}, 1 \mathrm{~ns}$ and $25 \mathrm{~ns}$ after excitation. These snapshots show directly how in this model the local order parameters $\psi_{m}$ evolve by randomizing their phase $\varphi_{m}$ around a ring at constant $\left|\psi_{m}\right|$ until the metallic state at $\left|\left\langle\psi_{m}\right\rangle\right|=0$ is reached.

In this context, questions remain about the microscopic origin of the metastable light-induced superconducting state. From the observation that the amplitude of the effect is dependent only on the integrated pulse area, it becomes clear that, at least in the long-pulse regime, the previously proposed non-linear phonon mechanism ${ }^{6}$, which was suggested to generate a displaced crystal structure, is most likely not correct. If it were correct, one would expect a response that depends on some power of the electric field, rather than on only the total energy of the pulse. That said, mechanisms based on the parametric coupling of the light field to the electronic properties, either to amplify a superconducting order parameter ${ }^{31-33}$ or to cool selected degrees of freedom ${ }^{34,35}$, are not necessarily inconsistent with these observations.

Our findings prompt comparison with previously measured responses that were observed under sustained driving, such as the microwave enhancement of conventional superconductivity $^{36,37}$. One evident difference with these measurements is that the temperature scale observed here is far larger than that reported in the microwave-enhancement case. Furthermore, in the case of microwave-enhanced Bardeen-Cooper-Schrieffer superconductivity, the effect was observed only for excitation below the superconducting 
a
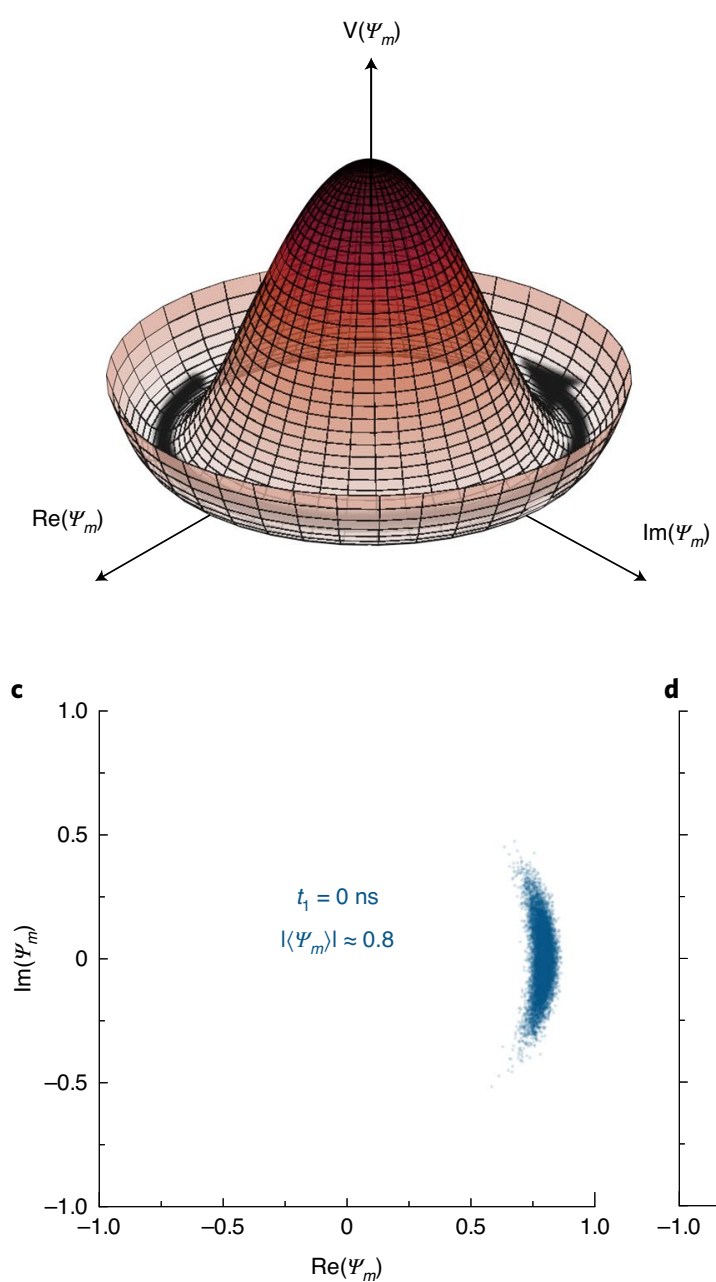

b
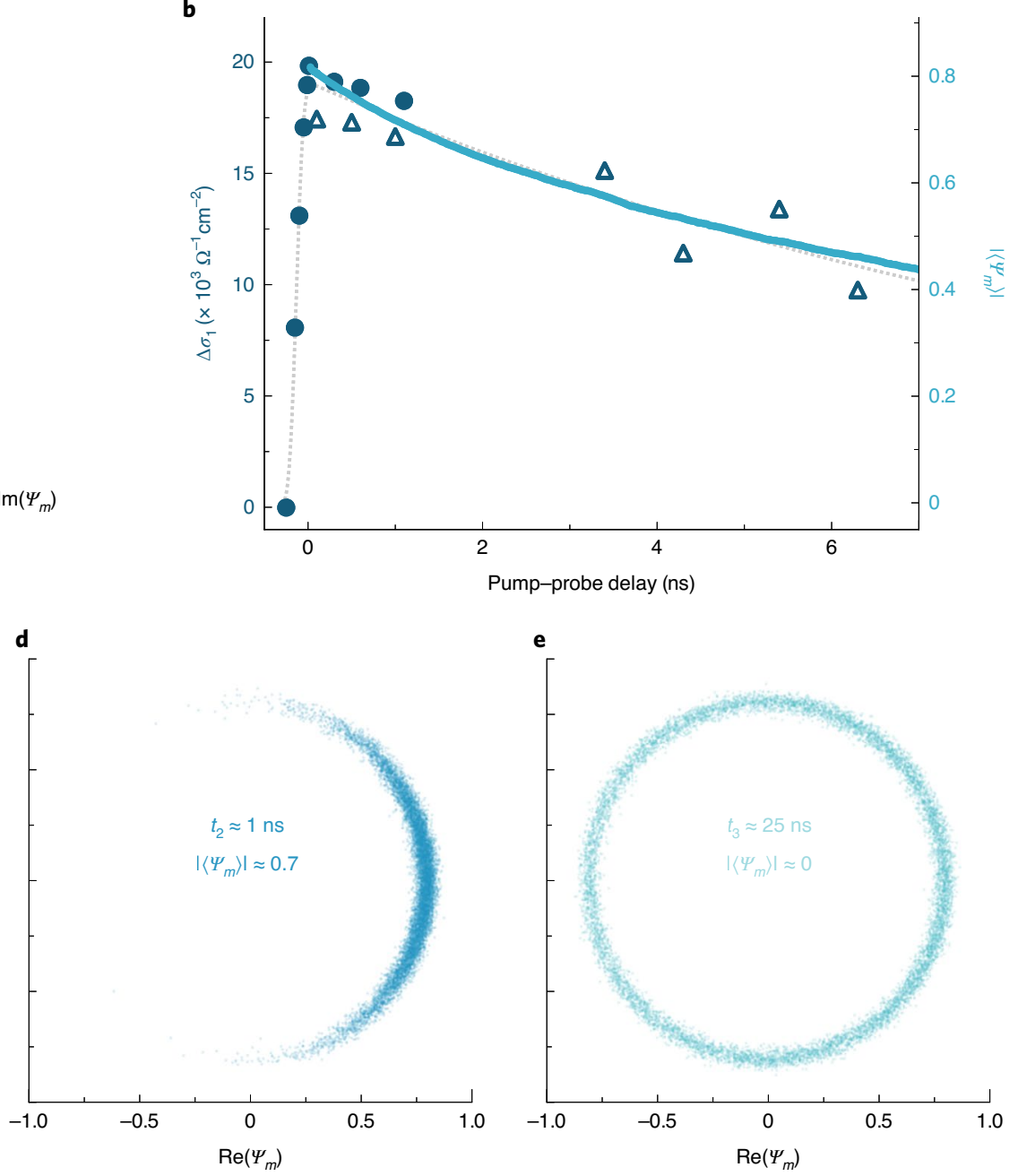

Fig. 6 | Modelling of the relaxation of the superconducting order parameter. a, Local free energy potential as a function of the local complex superconducting order parameter $\psi_{m}$. The arrow indicates the phase diffusion channel. $\mathbf{b}$, Time evolution of the integrated loss of spectral weight in the region of the superconducting gap (left axis, blue dots and triangles denote measurements on two different samples) and corresponding modelling (right axis, solid blue curve) using a time-dependent Ginzburg-Landau framework as described in the main text. The grey dotted line is a guide to the eye. c-e, Snapshots of the on-site complex order parameters $\psi_{m}$ for three different time delays of 0 ns (c) (right after excitation), $1 \mathrm{~ns}$ (d) and $25 \mathrm{~ns}$ (e).

gap, which was interpreted to be a result of quasi-particle redistribution and of renormalization of the parameters entering the BardeenCooper-Schrieffer equations ${ }^{38}$. In the microwave experiments, complementary measurements, in which excitation was tuned immediately above the gap, yielded a reduction of the superconducting order. Here, our experiments are conducted in a different regime; that is, at pump-photon energies that are at least one order of magnitude larger than the low-temperature equilibrium superconducting gap, and in a regime in which the primary coupling of the mid-infrared radiation is not with the condensate, but with other high-energy excitations, such as molecular vibrations or collective electronic modes. Hence, it is unlikely that the mechanism invoked for microwave enhancement can explain these observations.

Other experimental reports have documented a sustained, metastable enhancement of superconductivity in oxygen-deficient $\mathrm{YBa}_{2} \mathrm{Cu}_{3} \mathrm{O}_{7-\delta}$ samples after exposure to radiation with frequencies ranging from the ultraviolet to the near-infrared ${ }^{39-41}$. However, in all of these cases, the superconducting transition temperature of the irradiated superconductor never exceeded the equilibrium transition temperature at optimal doping. These observations were either interpreted as a result of photodoping towards a more metallic state favouring superconductivity, or involved annealing of oxygen-deficient samples. By contrast, the data reported here show enhanced superconducting properties well above the highest equilibrium transition temperature observed in any one of the $\mathrm{A}_{3} \mathrm{C}_{60}$ compounds ${ }^{13}$, by excitation with photon energies away from inter-band transitions. Therefore, none of the previously invoked mechanisms offers a plausible explanation for our findings.

The discovery of a metastable light-induced state with clear signatures of superconductivity holds compelling promise in the quest to extend lifetimes even further. New lasers that are capable of generating longer pulses or suitably designed trains of pulses could be developed to sustain the coherence of this state. Extended lifetimes will also open up the possibility of studying these effects with other low-frequency probes, which range from measurements of magnetic susceptibility to scattering and transport methods. Recent theoretical ${ }^{42}$ and experimental ${ }^{43}$ reports suggest that the superconducting order parameter can be influenced also by the electromagnetic environment of an optical cavity. Indeed, observations made in alkali-doped fullerides ${ }^{43}$ could be expanded on by combining cavity settings with external driving ${ }^{44}$ as a means to reduce the required excitation and hence dissipation and heating. 


\section{Online content}

Any methods, additional references, Nature Research reporting summaries, source data, extended data, supplementary information, acknowledgements, peer review information; details of author contributions and competing interests; and statements of data and code availability are available at https://doi.org/10.1038/ s41567-020-01148-1.

Received: 27 June 2020; Accepted: 11 December 2020; Published online: 4 February 2021

\section{References}

1. Nova, T. F., Disa, A. S., Fechner, M. \& Cavalleri, A. Metastable ferroelectricity in optically strained $\mathrm{SrTiO}_{3}$. Science 364, 1075-1079 (2019).

2. $\mathrm{Li}, \mathrm{X}$. et al. Terahertz field-induced ferroelectricity in quantum paraelectric $\mathrm{SrTiO}_{3}$. Science 364, 1079-1082 (2019).

3. Disa, A. S. et al. Polarizing an antiferromagnet by optical engineering of the crystal field. Nat. Phys. 16, 937-941 (2020).

4. Fausti, D. et al. Light-induced superconductivity in a stripe-ordered cuprate. Science 331, 189-191 (2011).

5. $\mathrm{Hu}, \mathrm{W}$. et al. Optically enhanced coherent transport in $\mathrm{YBa}_{2} \mathrm{Cu}_{3} \mathrm{O}_{6.5}$ by ultrafast redistribution of interlayer coupling. Nat. Mater. 13, 705-711 (2014).

6. Mitrano, M. et al. Possible light-induced superconductivity in $\mathrm{K}_{3} \mathrm{C}_{60}$ at high temperature. Nature 530, 461-464 (2016).

7. Cantaluppi, A. et al. Pressure tuning of light-induced superconductivity in $\mathrm{K}_{3} \mathrm{C}_{60}$. Nat. Phys. 14, 837-841 (2018).

8. Buzzi, M. et al. Photo-molecular high temperature superconductivity. Phys. Rev. X 10, 031028 (2020).

9. Liu, B. et al. Pump frequency resonances for light-induced incipient superconductivity in $\mathrm{YBa}_{2} \mathrm{Cu}_{3} \mathrm{O}_{6.5}$. Phys. Rev. X 10, 011053 (2020).

10. Hebard, A. F. et al. Superconductivity at $18 \mathrm{~K}$ in potassium-doped $\mathrm{C}_{60}$. Nature 350, 600-601 (1991).

11. Xiang, X.-D. et al. Synthesis and electronic transport of single crystal $\mathrm{K}_{3} \mathrm{C}_{60}$. Science 256, 1190-1191 (1992).

12. Gunnarsson, O. Alkali-doped Fullerides: Narrow-band Solids with Unusual Properties (World Scientific, 2004).

13. Ganin, A. Y. et al. Polymorphism control of superconductivity and magnetism in $\mathrm{Cs}_{3} \mathrm{C}_{60}$ close to the Mott transition. Nature 466, 221-225 (2010).

14. Takabayashi, Y. et al. The disorder-free non-BCS superconductor $\mathrm{Cs}_{3} \mathrm{C}_{60}$ emerges from an antiferromagnetic insulator parent state. Science 323, 1585-1590 (2009).

15. Takabayashi, Y. \& Prassides, K. Unconventional high- $T_{\mathrm{c}}$ superconductivity in fullerides. Phil. Trans. R. Soc. A 374, 20150320 (2016).

16. Nicoletti, D. et al. Optically induced superconductivity in striped $\mathrm{La}_{2-x} \mathrm{Ba}_{x} \mathrm{CuO}_{4}$ by polarization-selective excitation in the near infrared. Phys. Rev. B 90, 100503 (2014).

17. Cremin, K. A. et al. Photoenhanced metastable $c$-axis electrodynamics in stripe-ordered cuprate $\mathrm{La}_{1.885} \mathrm{Ba}_{0.115} \mathrm{CuO}_{4}$. Proc. Natl Acad. Sci. USA 116, 19875 (2019).

18. Nicoletti, D., Mitrano, M., Cantaluppi, A. \& Cavalleri, A. Comment on 'Terahertz time-domain spectroscopy of transient metallic and superconducting states'. Preprint at https://arxiv.org/abs/1506.07846 (2015).

19. Orenstein, J. \& Dodge, J. S. Terahertz time-domain spectroscopy of transient metallic and superconducting states. Phys. Rev. B 92, 134507 (2015).

20. Babzien, M., Pogorelsky, I. V. \& Polanskiy, M. Solid-state seeding of a high power picosecond carbon dioxide laser. AIP Conf. Proc. 1777, 110001 (2016).

21. Alcock, A. J. \& Corkum, P. B. Ultra-short pulse generation with $\mathrm{CO}_{2}$ lasers. Phil. Trans. R. Soc. Lond. Ser. A 298, 365-376 (1980).

22. Alcock, A. J., Corkum, P. B. \& James, D. J. A fast scalable switching technique for high-power $\mathrm{CO}_{2}$ laser radiation. Appl. Phys. Lett. 27, 680-682 (1975).

23. Mayer, B. et al. Sub-cycle slicing of phase-locked and intense mid-infrared transients. New J. Phys. 16, 063033 (2014).

24. Waterman, A. T. An equilibrium theory of electrical conduction. Phys. Rev. 22, 259-270 (1923).
25. Bartlett, R. S. Photo-resistance effect for metals at low temperatures. Phys. Rev. 26, 247-255 (1925).

26. Wilson, T. C. Photoconductivity of metal films. Phys. Rev. 55, 316-317 (1939).

27. Heyman, J. N. et al. Carrier heating and negative photoconductivity in graphene. J. Appl. Phys. 117, 015101 (2015).

28. Uemura, Y. J. Dynamic superconductivity responses in photoexcited optical conductivity and Nernst effect. Phys. Rev. Mater. 3, 104801 (2019).

29. Lucas, G. \& Stephen, M. J. Relaxation of the superconducting order parameter. Phys. Rev. 154, 349-353 (1967).

30. Madan, I. et al. Nonequilibrium optical control of dynamical states in superconducting nanowire circuits. Sci. Adv. 4, eaao0043 (2018)

31. Babadi, M., Knap, M., Martin, I., Refael, G. \& Demler, E. Theory of parametrically amplified electron-phonon superconductivity. Phys. Rev. B 96, 014512 (2017).

32. Knap, M., Babadi, M., Refael, G., Martin, I. \& Demler, E. Dynamical Cooper pairing in nonequilibrium electron-phonon systems. Phys. Rev. B 94, 214504 (2016).

33. von Hoegen, A. et al. Parametrically amplified phase-incoherent superconductivity in $\mathrm{YBa}_{2} \mathrm{Cu}_{3} \mathrm{O}_{6+x}$. Preprint at https://arxiv.org/ abs/1911.08284 (2020).

34. Denny, S. J., Clark, S. R., Laplace, Y., Cavalleri, A. \& Jaksch, D. Proposed parametric cooling of bilayer cuprate superconductors by terahertz excitation. Phys. Rev. Lett. 114, 137001 (2015).

35. Nava, A., Giannetti, C., Georges, A., Tosatti, E. \& Fabrizio, M. Cooling quasiparticles in $\mathrm{A}_{3} \mathrm{C}_{60}$ fullerides by excitonic mid-infrared absorption. Nat. Phys. 14, 154-159 (2018).

36. Anderson, P. W. \& Dayem, A. H. Radio-frequency effects in superconducting thin film bridges. Phys. Rev. Lett. 13, 195-197 (1964).

37. Wyatt, A. F. G., Dmitriev, V. M., Moore, W. S. \& Sheard, F. W. Microwave-enhanced critical supercurrents in constricted tin films. Phys. Rev. Lett. 16, 1166-1169 (1966).

38. Eliashberg, G. \& Film, M. Superconductivity stimulated by a high-frequency field. JETP Lett. 11, 114-116 (1970).

39. Yu, G., Heeger, A. J., Stucky, G., Herron, N. \& McCarron, E. M. Transient photoinduced conductivity in semiconducting single crystals of $\mathrm{YBa}_{2} \mathrm{Cu}_{3} \mathrm{O}_{6.3}$ : search for photoinduced metallic state and for photoinduced superconductivity. Solid State Commun. 72, 345-349 (1989).

40. Yu, G. et al. Phase separation of photogenerated carriers and photoinduced superconductivity in high- $T_{\mathrm{c}}$ materials. Phys. Rev. B 45, 4964-4977 (1992).

41. Nieva, G. et al. Photoinduced enhancement of superconductivity. Appl. Phys. Lett. 60, 2159-2161 (1992).

42. Schlawin, F., Cavalleri, A. \& Jaksch, D. Cavity-mediated electron-photon superconductivity. Phys. Rev. Lett. 122, 133602 (2019).

43. Thomas, A. et al. Exploring superconductivity under strong coupling with the vacuum electromagnetic field. Preprint at https://arxiv.org/abs/1911.01459 (2019).

44. Gao, H., Schlawin, F., Buzzi, M., Cavalleri, A. \& Jaksch, D. Photo-induced electron pairing in a driven cavity. Phys. Rev. Lett. 125, 053602 (2020).

Publisher's note Springer Nature remains neutral with regard to jurisdictional claims in published maps and institutional affiliations.

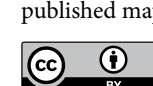

Open Access This article is licensed under a Creative Commons

Attribution 4.0 International License, which permits use, sharing, adaptation, distribution and reproduction in any medium or format, as long as you give appropriate credit to the original author(s) and the source, provide a link to the Creative Commons license, and indicate if changes were made. The images or other third party material in this article are included in the article's Creative Commons license, unless indicated otherwise in a credit line to the material. If material is not included in the article's Creative Commons license and your intended use is not permitted by statutory regulation or exceeds the permitted use, you will need to obtain permission directly from the copyright holder. To view a copy of this license, visit http://creativecommons. org/licenses/by/4.0/.

(c) The Author(s), under exclusive licence to Springer Nature Limited 2021 


\section{Methods}

All methods can be found in the Supplementary Information.

\section{Data availability}

Source data are provided with this paper. All other data that support the plots within this paper and other findings of this study are available from the corresponding authors on reasonable request.

\section{Acknowledgements}

The research leading to these results received funding from the European Research Council under the European Union's Seventh Framework Programme (FP7/2007-2013)/ ERC grant agreement no. 319286 (QMAC). We acknowledge support from the Deutsche Forschungsgemeinschaft via the Cluster of Excellence 'The Hamburg Centre for Ultrafast Imaging' (EXC 1074 - project ID 194651731). E.W. was supported by a fellowship from the Alexander von Humboldt Foundation. We thank M. Volkmann for his technical assistance in the construction of the new optical apparatus presented in this work. We are also grateful to E. König, B. Fiedler and B. Höhling for their support in the fabrication of the electronic transport samples, and to J. Harms for assistance with graphics. Open access funding was provided by the Max Planck Society.

\section{Author contributions}

M. Budden, T.G., M. Buzzi, G.J., G.M., Y.L. and A.C. conceived the experiment. A.C. supervised the project. The set-up shown in Fig. 2 was built and related measurements were performed by M. Buzzi and G.J. The set-up shown in Fig. 3 was developed by M. Budden and T.G. with support of Y.L. and related measurements were performed by M. Budden and T.G. Data analysis of the optical measurements was conducted by M. Budden, T.G., M. Buzzi and G.J. Transport measurements (Figs. 4 and 5) were performed by M. Budden and T.G. with support by T.M. and G.M. Preparation of the samples for electronic transport was carried out by M. Budden, T.G. and E.W. Custom measurement electronics and circuit simulations were performed by T.M. and G.M. $\mathrm{K}_{3} \mathrm{C}_{60}$ samples were provided by D.P. and M.R. The Ginzburg-Landau model of the superconducting order parameter relaxation was developed by F.S. and D.J. The manuscript was written by M. Budden, M. Buzzi, T.G., T.M., G.M. and A.C. with contributions from all other authors.

\section{Competing interests}

The authors declare no competing interests.

\section{Additional information}

Supplementary information is available for this paper at https://doi.org/10.1038/ s41567-020-01148-1.

Correspondence and requests for materials should be addressed to A.C. or M.B.

Peer review information Nature Physics thanks Giulio Cerullo and the other, anonymous, reviewer(s) for their contribution to the peer review of this work.

Reprints and permissions information is available at www.nature.com/reprints. 